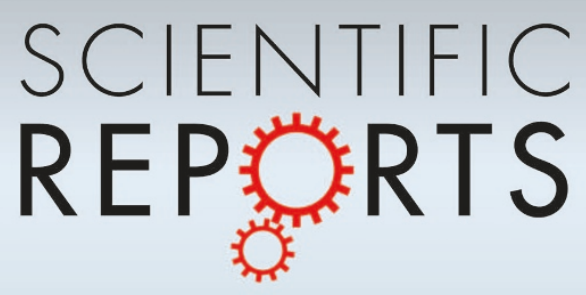

\title{
OPEN Release of Cyclic Phosphatidic Acid from Gelatin-based Hydrogels Inhibit Colon \\ SUBJECT AREAS: \\ LIPIDS Cancer Cell Growth and Migration
}

CELL GROWTH

TARGET IDENTIFICATION

CANCER MODELS

Received

25 July 2012

Accepted

6 September 2012

Published

24 September 2012

Correspondence and requests for materials should be addressed to T.T. (ttamotsu@shinshuu.ac.jp)

\author{
Tamotsu Tsukahara' \& Kimiko Murakami-Murofushi ${ }^{2}$
}

\begin{abstract}
'Department of Integrative Physiology \& Bio-System Control, Shinshu University School of Medicine, 3-1-1 Asahi, Matsumoto, Nagano 390-862 1, Japan, ${ }^{2}$ Department of Biology, Faculty of Science, Ochanomizu University, 2-1-1 Ohtsuka, Bunkyo-ku, Tokyo 112-8610, Japan.
\end{abstract}

Microparticle and nanoparticle formulations are widely used to improve the bioavailability of low-solubility drugs and as vehicles for organ- and tissue-specific targeted drug delivery. We investigated the effect of a novel, controlled-release form of a bioactive lipid, cyclic phosphatidic acid (cPA), on human colon cancer cell line functions. We encapsulated cPA in gelatin-based hydrogels and examined its ability to inhibit the viability and migration of HT-29 and DLD-1 cells in vitro and the LPA-induced activity of the transcription factor peroxisome proliferator-activated receptor gamma (PPAR $\gamma$ ). The hydrogel delivery system prolonged cPA release into the culture medium. Accordingly, cPA-hydrogel microspheres substantially inhibited LPA-induced PPAR $\gamma$ activity and cell growth and migration compared with that of cells cultured with cPA alone. Thus, hydrogel microspheres are a potential system for stable and efficient delivery of bioactive lipids such as $\mathrm{CPA}$ and may offer a new strategy for targeted colon cancer treatment.

M etastatic colon cancer is associated with high mortality ${ }^{1}$. Although the outcome of advanced colon cancer has been improved by combining the first-line chemotherapy agent 5-fluorouracil with new drugs such as bevacizumab and cetuximab ${ }^{2,3}$, the prognosis for metastatic colon cancer remains very poor, and novel therapeutic strategies are needed to reduce the mortality of this disease. Recent reports suggest that peroxisome proliferator-activated receptor $\gamma(\operatorname{PPAR} \gamma)$ is an important molecular target for the development of anticancer treatments ${ }^{4-6}$. PPAR $\gamma$ is a member of a family of nuclear transcription factors that regulate the expression of genes controlling a number of key metabolic processes, including lipid and glucose homeostasis ${ }^{7,8}$, cell proliferation ${ }^{9}$, and inflammation ${ }^{10}$. The PPARs are activated by fatty acids, leukotrienes, and prostaglandins, and these molecules initiate the transcription of an array of genes involved in energy homeostasis. Upon ligand binding, PPAR $\gamma$ heterodimerizes with the retinoid $\mathrm{X}$ receptor, $\mathrm{RXR} \alpha$, and the heterodimer then recruits additional proteins such as coactivators (SRC-1, CBP, and TRAP220) and corepressors (SMRT and NCoR) to modulate gene transcription ${ }^{11}$. Although PPAR $\gamma$ is highly expressed in the mucosa of the colon and rectum ${ }^{12}$, the potential benefits of modulating PPAR $\gamma$ in colon cancer remain controversial. We recently reported that PPAR $\gamma$ is required for the growth inhibition of colon cancer cells induced by cyclic phosphatidic acid (cPA), a naturally occurring structural analog of lysophosphatidic acid (LPA) ${ }^{13}$. cPA is known to be generated via phospholipase D-catalysed transphosphatidylation of $\mathrm{LPC}^{14}$ and has been shown to be present in plasma ${ }^{15}$. cPA is a stable lipid, with $75 \%$ of the molecule remaining intact after incubation in tissue culture medium for up to $24 \mathrm{~h}^{16,17}$. cPA can be converted to LPA by opening the ring structure at the $s n-2$ and $s n-3$ positions of the glycerol backbone via the action of endogenous and exogenous phosphatases ${ }^{18,19}$.

Bioactive lipids, particularly lysophospholipids, are a group of molecules that have multiple biological functions, including regulation of cell proliferation, morphology, and migration. One of the most bioactive lipids is water-soluble LPA, which has been previously shown to enhance cancer cell proliferation, migration, and survival $^{20}$. LPA was initially found at considerable levels in the ascites of ovarian cancer patients ${ }^{21}$. However, it has recently been reported that LPA levels are increased not only in the body fluid of ovarian cancer patients, but also in the plasma of patients with colon $\operatorname{cancer}^{22,23}$. These reports suggest that LPA also plays important roles in the progression of colon cancer. These cellular effects are elicited through cell surface G protein-coupled receptors $^{24-27}$; and can also directly interact with intracellular targets such as PPAR $\gamma^{28-30}$. To date, 7 LPA-specific cell surface receptors have been identified. $\mathrm{CPA}$ is a weak agonist of many LPA receptors ${ }^{31}$, and it has been reported that $\mathrm{CPA}$ has antimitogenic effects ${ }^{32}$ and prevents cancer cell invasion in vitro ${ }^{33}$ and metastasis in vivo ${ }^{34,18}$. $\mathrm{cPA}$ 
a

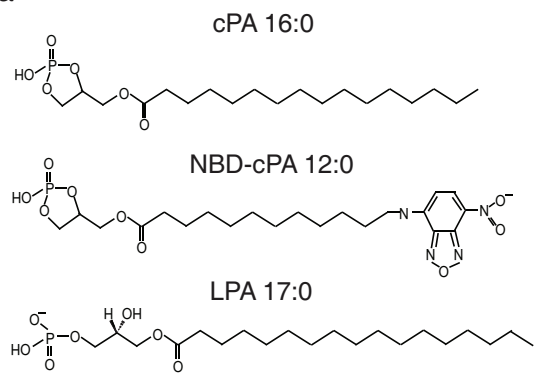

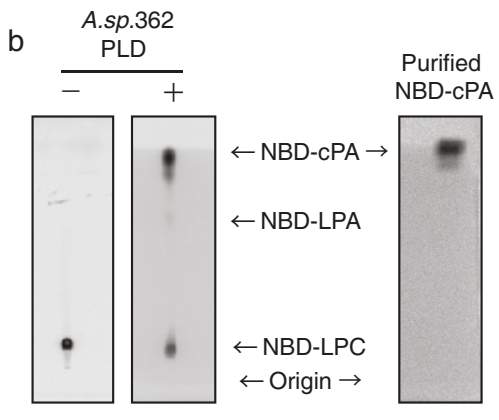

Fluorescence
Light
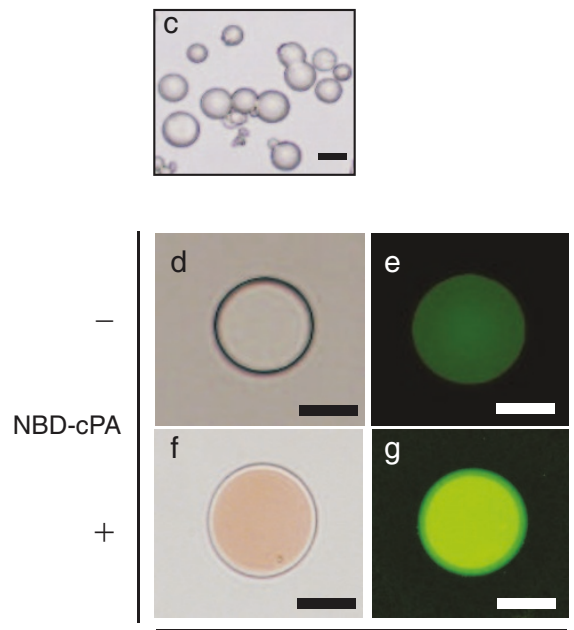

Hydrogel h
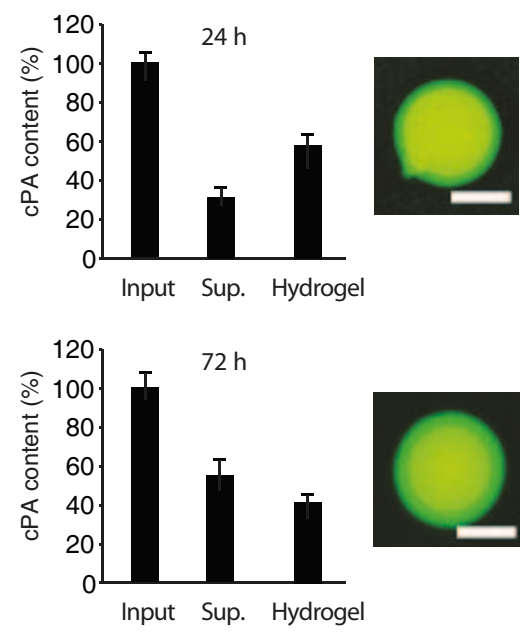

Figure 1 Visualization of hydrogel-encapsulated lipids by fluorescence microscopy and ultraviolet light. (a) Structural formulae of compounds examined in this study. (b) Efficient synthesis of NBD-cPA from NBD-LPC using Actinomadura PLD. (c) Light micrograph of pure microspheres in the swollen state, scale bar $=80 \mu \mathrm{m}$. (d-g) Buffer diffusion into an NBD-labelled hydrogel was monitored by confocal microscopy. Light and fluorescent microscopic images of hydrogel microspheres loaded with NBD-cPA. Scale bar $=50 \mu \mathrm{m}$. (h) Distribution of cPA-hydrogel microspheres in the growth medium after 24 and $72 \mathrm{~h}$ of incubation. Scale bar $=50 \mu \mathrm{m}$. NBD-cPA from the supernatant fractions (Sup.) or NBD-cPA incorporated in microspheres (Hydrogel) was then quantified by the molybdenum blue method. The data is shown as a percentage of the input NBD-cPA present in supernatants and NBD-cPA microspheres.

inhibits the LPA-induced invasion of many cancer cell lines by 70$95 \%{ }^{16,33}$. Furthermore, cPA inhibits LPA-induced RhoA activation, suggesting that the anti-metastatic effect of $\mathrm{cPA}$ is achieved by the inhibition of RhoA activation ${ }^{35}$. These reports suggest that the cellular effects of cPA are remarkably different from those of LPA.

Recently, biocompatible hydrogels and delivery methods have been developed with the aim of achieving controlled release of therapeutic drugs ${ }^{36}$. Hydrogels are thermodynamically compatible with water, which allows them to swell in aqueous media ${ }^{37}$. The hydrogels form a reservoir-based, swelling-controlled, release system in which the drug is incorporated into the hydrophilic hydrogel matrix, and diffuses out in a controlled fashion as the hydrogel swells. One of the newest approaches to drug delivery uses gelatin-based hydrogels ${ }^{38}$. Gelatin is a natural, biodegradable, non-toxic macromolecule, and these properties have led to its wide application in drug delivery. In this study, we formulated cPA-containing gelatin hydrogels, and we showed that binding within the hydrogel microspheres is essential to prevent degradation of $\mathrm{CPA}$ and maintain long-term biological activity in the aqueous environment.

\section{Results}

Synthesis and analysis of fluorescent NBD-cPA. Fluorescent 7-nitro2-1,3-benzoxadiazole-4-yl (NBD)-labelled lipids are commonly used as analogs of native lipids, and closely related probes have been used to monitor the intracellular localization and spatial dynamics of lipid molecules $^{39}$. In this study, we synthesised NBD-labelled cPA from NBD-tagged lysophosphatidylcholine (LPC) (Fig. 1a). As shown in Fig. $1 \mathrm{~b}$, reaction progress was monitored by the appearance of a cPA spot on thin layer chromatography (TLC), visualised by fluorescence imaging (Fig. 1b) and $0.01 \%$ primuline staining (data not shown). The TLC analysis confirmed the purity of authentic NBDcPA. In a chloroform:methanol:28\% ammonium hydroxide solvent (6:4:1), purified NBD-cPA had with a retention factor $(\mathrm{R} f)$ value of 0.92 , whereas NBD-LPC had with an R $f$ of 0.14 . Murakami-Murofushi et al. ${ }^{18}$ examined the formation of CPA from LPC by phospholipase D (PLD) derived from Actinomadura sp. No. 362, and found that under certain conditions, this enzyme preferentially catalyzes the transphosphatidylation of LPC to CPA rather than hydrolysis to LPA, and thus produces $\mathrm{cPA}$ almost exclusively (Fig. $1 \mathrm{~b})^{18}$. We found the procedure described here to be useful for the purification of NBD-cPA from the reaction mixture.

Characterization of hydrogel microspheres. Fig. 1c shows a typical light micrograph of the hydrogel microspheres before loading with NBD-cPA in PBS. The red colour of the NBD-cPA surrounding the microspheres in Fig. If becomes lighter after incorporation of $\mathrm{NBD}-\mathrm{cPA}$ into the core of the hydrogel microsphere. NBD-cPA 
a

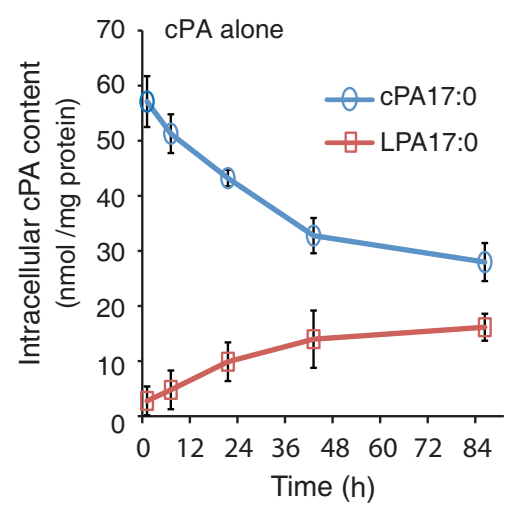

b

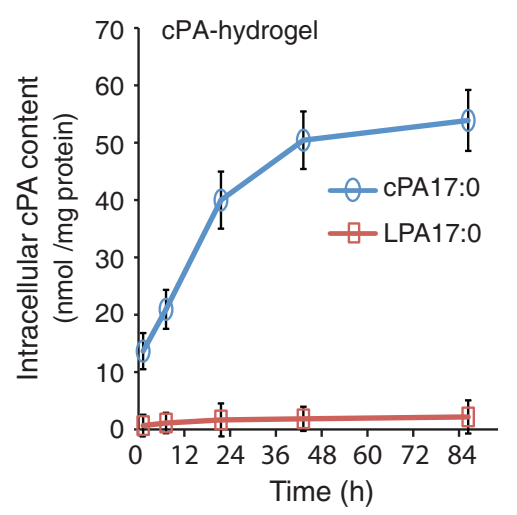

C

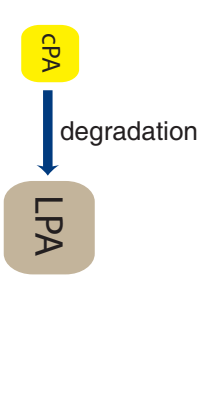

(medium)

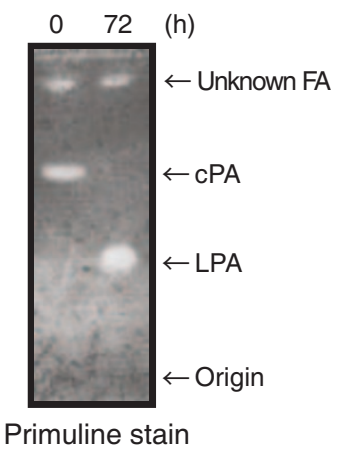

d

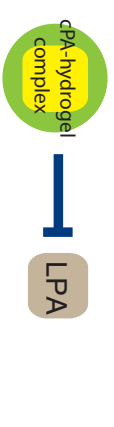

(medium)

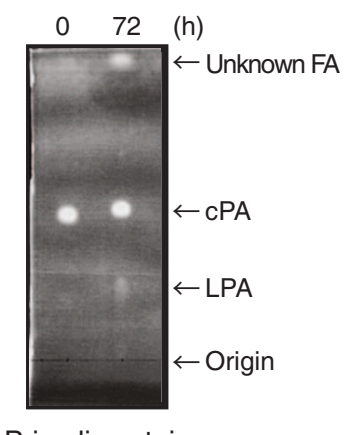

Primuline stain
cPA degradation

Figure $2 \mid$ Intracellular cPA content from gelatin hydrogels in vitro. (a and b) Time course of the degradation of cPA to LPA (a), or the release of adsorbed cPA from hydrogel microspheres (b) in HT-29 cultures at $37^{\circ} \mathrm{C}$. Cells were harvested by scraping, and lipids were extracted using the method reported by Bligh and Dyer. The extracted lipids were dissolved in $0.1 \mathrm{~mL}$ of methanol:chloroform:28\% $\mathrm{NH}_{4} \mathrm{OH}(90: 10: 1)$ and were immediately analysed by ESI-LC-MS. (c and d) TLC analysis of degradation products from cPA alone (c) or cPA-hydrogel microspheres (d) in the medium after $72 \mathrm{~h}$ of incubation. Conditioned medium was harvested and extracted in chloroform:methanol (1:1) solvent system. Lipids were extracted from the cells, separated by thin-layer chromatography, and visualised with $0.1 \%$ primuline.

distribution (up to $72 \mathrm{~h}$ ) can also be visualised by fluorescence microscopy, which shows NBD-cPA fluorescing strongly in the centre of the hydrogel microsphere (Fig. $1 \mathrm{~g}$ and h; excitation $470 \mathrm{~nm} /$ emission $530 \mathrm{~nm}$ ). This finding confirmed that NBD-cPA had successfully penetrated the microsphere surface. To investigate further, we examined the release of cPA 17:0 (a chemically synthesised, non-physiological cPA) from the hydrogel microspheres by electrospray ionization liquid chromatography-mass spectrometry (ESI-LC-MS) and TLC analysis. In Fig. 2a and b, HT-29 cells were incubated with free cPA (Fig. 2a) or cPA-hydrogel (Fig. 2b) at $37^{\circ} \mathrm{C}$. We previously reported that $\mathrm{CPA}$ inhibits the growth of colon cancer cells $^{13}$, which raised the possibility of using cPA for cancer therapy. However, hydrolytic cleavage of the cyclic phosphate ring of cPA by lipid phosphate phosphatases leads to the formation of LPA, which is a growth stimulator ${ }^{40}$. Indeed, in long-term cultures of HT-29 cells incubated with cPA alone, we observed the production of LPA (Fig. 2a). TLC analysis (Fig. 2c) after $72 \mathrm{~h}$ of incubation confirmed that $>85 \%$ of cPA was converted into LPA and an unknown fatty acid (FA). In contrast, when HT-29 cells were treated with cPA-hydrogel microspheres, the $\mathrm{cPA}$ was released into the culture medium, but almost no conversion of cPA to LPA was observed (Fig. 2d). These results suggest that $\mathrm{CPA}$ bound within the hydrogel microspheres was protected from degradation during long-term incubation $(>72 \mathrm{~h})$ in cell culture medium.

cPA-hydrogel microspheres inhibit LPA-induced activation of PPAR $\gamma$. We next examined the ability of cPA-hydrogel microspheres to inhibit LPA-induced PPAR $\gamma$ activation in HT-29 and DLD-1 cells. Our previous work showed that the cPA-induced growth inhibition of HT-29 cells is mediated by inhibition of the PPAR $\gamma$ pathway ${ }^{13}$; therefore, we investigated whether the same effect could be observed with cPA-hydrogel microspheres. For this purpose, HT-29 and DLD- 1 cells were plated in 96-well plates $\left(5 \times 10^{3} /\right.$ well $)$ in DMEM supplemented with $10 \%$ FBS. The next day, cells were transiently transfected with a luciferase reporter $(125 \mathrm{ng})$ containing 3 copies of PPRE driven by the promoter for acyl CoA oxidase (pGL3PPRE-acyl-CoA oxidase-luciferase), pcDNA3.1-PPAR $\gamma$ (62.5 ng), and pSV- $\beta$-galactosidase (12.5 ng) (Promega) using Lipofectamine 2000 (Invitrogen). Transfected cells were incubated for $20 \mathrm{~h}$ or $48 \mathrm{~h}$ with combinations of vehicle, free cPA, cPA-hydrogel microspheres, and the PPAR $\gamma$ agonist LPA 18:1. As shown in Fig. 3a, luciferase activity increased upon the addition of 1 and $10 \mu \mathrm{M}$ LPA 18:1; however, as expected, no increase was observed in response to cPA alone or cPAhydrogel microspheres. In contrast, when $\mathrm{CPA}$, either alone or as CPAhydrogel microspheres, was added to cultures containing $10 \mu \mathrm{M}$ LPA 18:1, luciferase activity was inhibited, and cPA-hydrogel microspheres inhibited luciferase activity more strongly than did cPA alone, at both $20 \mathrm{~h}$ and $48 \mathrm{~h}$ of incubation. Therefore, LPA-induced PPAR $\gamma$ activity, as measured by luciferase reporter activity, was efficiently suppressed by co-treatment with cPA-hydrogel microspheres.

cPA-hydrogel microspheres inhibit the growth and migration of HT-29 and DLD-1 cells. To ensure that the hydrogel microspheres had no detrimental effects on cell function, we examined the viability, 
a

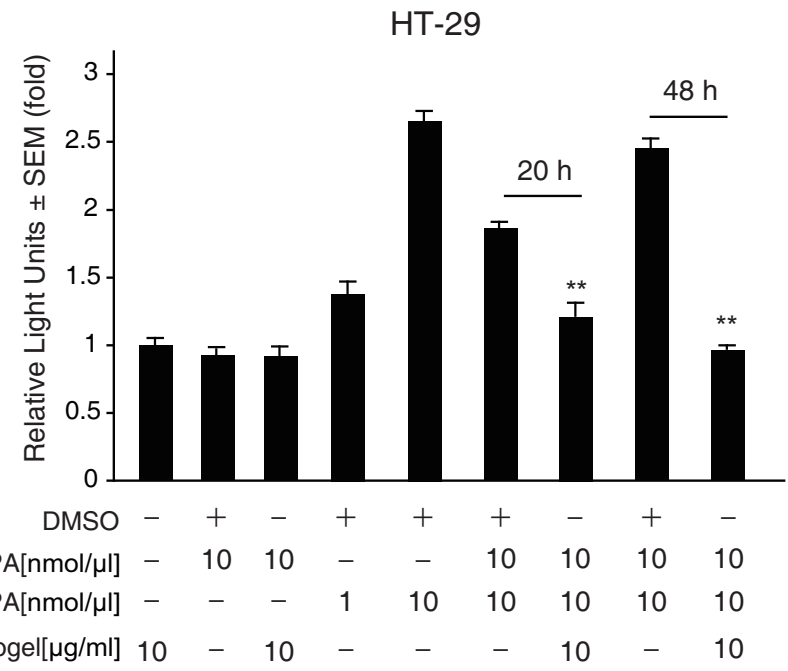

Hydrogel $[\mu \mathrm{g} / \mathrm{ml}] 10 \quad-10 \quad-\quad \ldots \quad-10 \quad-10$

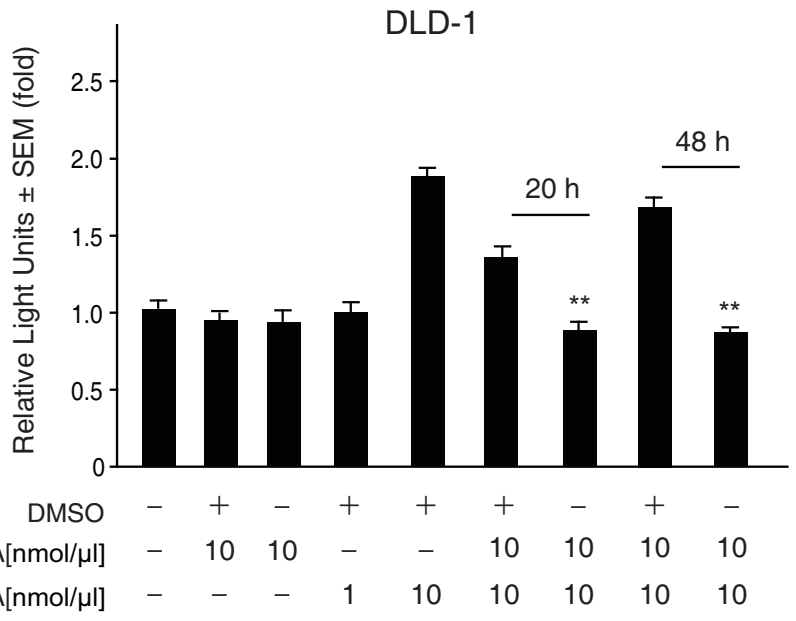

Hydrogel[$[\mathrm{\mu g} / \mathrm{ml}] \quad 10 \quad-10 \quad-\quad \ldots \quad-10 \quad-10$

Figure 3 Hydrogel microspheres protected cPA from degradation and suppress PPAR $\gamma$ activation. (a) HT-29 and DLD-1 cells were transfected with PPAR $\gamma$ expression and PPRE-luc reporter plasmids and treated with the vehicle (DMSO), cPA, LPA, cPA-hydrogel, or hydrogel microspheres for 20 or $48 \mathrm{~h}$. Luciferase activity was measured in the lysates of treated cells. Data are presented as mean \pm s.e.m.; $\mathrm{n}=3 ;{ }^{* *} \mathrm{P}<0.01$.

growth, and migration of HT-29 cells in the presence of control and cPA-containing hydrogel microspheres. As shown in Fig. 4a, the viability of cultured HT-29 and DLD-1 cells was not affected by incubation with various concentrations of control hydrogel microspheres (up to $30 \mu \mathrm{g} / \mathrm{mL}$ ). However, similar to the antitumor effects observed for CPA, the viability of HT-29 and DLD-1 cells decreased when incubated with $\mathrm{cPA}$, and the $\mathrm{CPA}$-hydrogel microspheres had a much greater effect on cell viability than $\mathrm{cPA}$ alone. Tumor cell migration across the endothelial barrier and penetration into the underlying basement membrane is an important step in cancer metastasis ${ }^{41}$. To investigate the potential anti-metastatic effects of cPA-hydrogel microspheres, we determined their effect on the migration of HT-29 cells in a wound-healing assay. A wound was inflicted in the HT-29 cell monolayers, and the widths were recorded (Fig. $4 \mathrm{~b}, \mathrm{t}=0$ ). Cells were then treated with vehicle or cPA-hydrogel microspheres, and photographs were taken over the next $72 \mathrm{~h}$ to measure wound widths. Representative results are shown in Fig. 4b and c, which demonstrate that HT-29 cell migration was substantially inhibited by incubation with the cPA-hydrogel microspheres. In Fig. 4d, a cell invasion assay using a modified Boyden chamber showed that co-treatment with cPA-hydrogel microspheres resulted in a substantial decrease in both HT-29 and DLD-1 cell invasion. Therefore, the cPA-hydrogel microspheres not only controlled the release and stability of cPA, but also inhibited HT-29 cell proliferation and migration, suggesting the cPA hydrogels might be useful for inhibiting metastasis. Our proposed mechanism of action for the cPA-hydrogel microspheres is summarised in Fig. 5a.

\section{Discussion}

Drug bioavailability is defined as the rate and extent at which the ingredients or active moiety is absorbed from the drug product and becomes available at the site of action. Ensuring adequate bioavailability is a key concern in drug development, particularly for poorly water-soluble drugs and novel entities for which there may be limited information on biodistribution. We prepared a resorbable bioactive lipid using a gelatin-based hydrogel, and evaluated its potential for controlled release of $\mathrm{cPA}$ in vitro. Hydrogels have many potential applications in the field of medicine, and biodegradable, injectable hydrogels might provide important delivery system options for bioactive molecules. We showed that the cPA-hydrogel microspheres inhibited the growth and migration of a colon cancer cell line, suggesting the potential use of cPA-hydrogel microspheres in the development of PPAR $\gamma$-targeted drug delivery. Our recent work identified $\mathrm{cPA}$ as a naturally occurring PPAR $\gamma$ antagonist $^{19,42}$, and exogenous cPA might also enter cells and inhibit PPAR $\gamma$ because cPA can bind to and displace the PPAR $\gamma$ agonist, rosiglitazone ${ }^{14}$. cPA suppresses PPAR $\gamma$ activation both by preventing the binding of exogenous agonists to PPAR $\gamma$ and inducing a specific conformational change that suppresses PPAR $\gamma$ activation. Using the hydrogel delivery system, cPA can be released for extended periods at $37^{\circ} \mathrm{C}$ while maintaining its structural and functional integrity. The complete resistance of $\mathrm{CPA}$ to phosphatase degradation was a key contributor to its stability following release from the hydrogel (Fig. 2c). We expect that novel preventive and therapeutic cancer treatments can be developed using cPA hydrogels that have a more favourable pharmacological impact than $\mathrm{CPA}$ alone. Over the past 2 decades, our understanding of the action of $\mathrm{CPA}$ has progressed to the point where it is now considered a potential drug candidate for cancers mediated through the PPAR $\gamma$ signalling pathway ${ }^{13}$. These studies have shown that cPA-hydrogel microspheres ensure steady and sustained release, which should improve bioavailability in vivo compared with cPA alone. Many pharmaceutical companies are exploiting the hydrogel technology to re-examine the potential of pharmacologically active molecules that were previously abandoned at the formulation stage because of poor solubility and bioavailability. Even though further research is needed on most biomolecule-containing hydrogels, including ligand-mediated delivery, they are likely to become important biomaterials in the near future. Therefore, it will be of great interest to confirm this novel anticancer strategy with further in vivo studies.

\section{Methods}

Reagents and antibodies. Biodegradable gelatin-based hydrogel (MedGel ${ }^{\circledR}$-PI5 microsphere; Kyoto, Japan) was purchased from WAKO Chemical (Tokyo, Japan). PLD from Actinomadura sp. No. 362 was purchased from Seikagaku Corporation (Tokyo, Japan). LPA (18:1) and fluorescent NBD-LPC were purchased from Avanti Polar Lipids Inc. (Alabaster, AL, USA). cPA 17:0 was chemically synthesised as previously described ${ }^{13}$. The purity of LPA and cPA was confirmed by TLC. LPA and cPA were quantified by the molybdenum blue method ${ }^{43}$ and stored under an argon atmosphere at $-85^{\circ} \mathrm{C}$ until use.

Plasmids and vectors. The pSV40- $\beta$-galactosidase and pcDNA3.1 plasmids were purchased from Promega (Madison, WI, USA) and Invitrogen (Carlsbad, CA, USA), respectively. The pcDNA3.1-PPAR $\gamma$ expression plasmid and pGL3b-PPRE-acylCoA oxidase-renilla luciferase (pGL3b-PPRE-Luc) reporter plasmid were constructed as previously described ${ }^{29}$. 
a

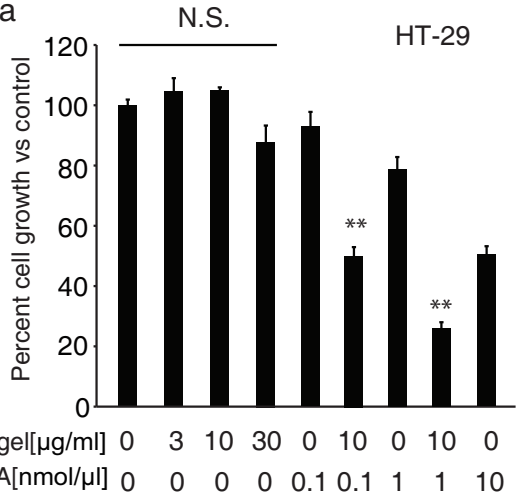

b

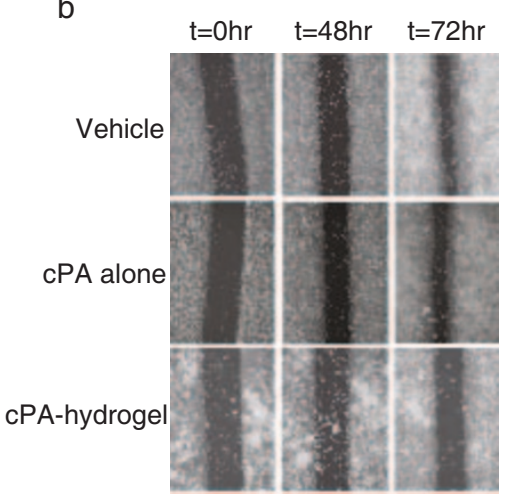

C

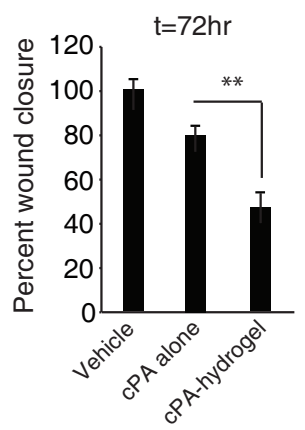

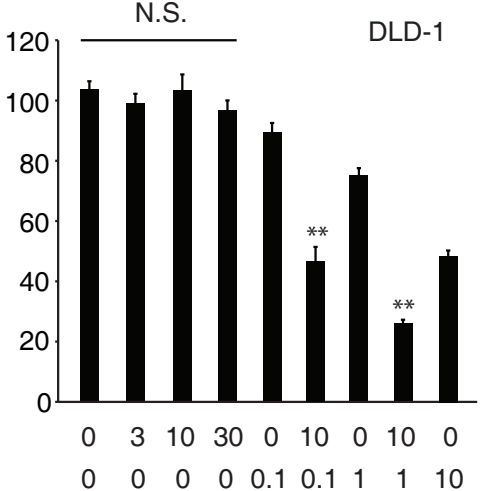

d

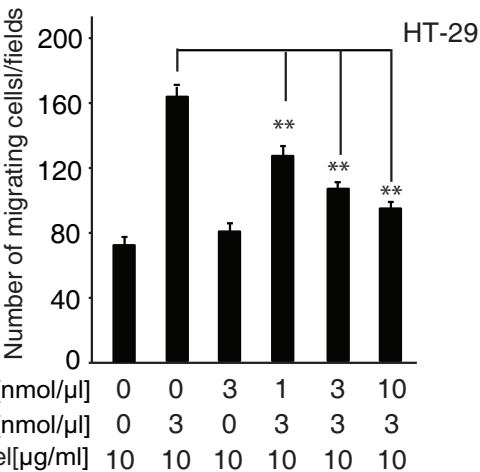

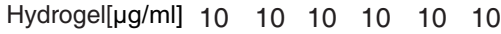

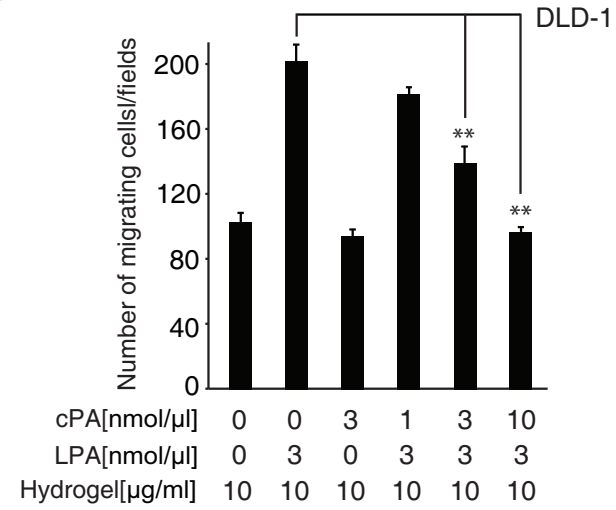

Figure $4 \mid$ Effect of cPA-hydrogel microspheres on viability and migration of HT-29 and DLD-1 cells. (a) Cells were treated with cPA hydrogels for 2 days, and cell viability and proliferation was then determined by alamarBlue assay. Data are presented as mean \pm s.e.m.; $n=4 ; * * P<0.01$. Similar results were obtained in 3 independent experiments. (b) Migration of HT-29 cells co-incubated with cPA or cPA-hydrogel microspheres was measured with a wound-healing assay. Images were taken after 0,48 , and $72 \mathrm{~h}$ of incubation using an Olympus IX70 microscope (100× magnification). (c) Data from the wound-healing assays are presented as mean \pm s.e.m.; $\mathrm{n}=3 ; * * \mathrm{P}<0.01$. Data are expressed as percentages of wound closure relative to the wound width at time 0 . Wound closure with vehicle-treated cells was regarded as $100 \%$. (d) Cells were seeded in 24-well plates containing a polycarbonate filter with 8 $\mu \mathrm{m}$ pores (Chemotaxicell CH8-24; Kurabo, Osaka, Japan) with $200 \mu \mathrm{L}$ of serum-free DMEM and incubated for $6 \mathrm{~h}$ at $37^{\circ} \mathrm{C}$ in the presence of $1 \mu \mathrm{M}$ LPA with or without $\mathrm{cPA}$ on the lower side of the filter. At least 4 fields per sample were counted and tabulated; values are expressed as mean (s.e.m.); $\mathrm{n}=4$; $* * \mathrm{P}<0.01$.

Cell culture and transfection. The human colon cancer cell line HT-29 was obtained from American Type Culture Collection (Manassas, VA, USA). DLD-1 human adenocarcinoma cells were obtained from Health Science Research Resources Bank (Osaka, Japan). Cells were grown in Dulbecco's modified Eagle's medium (DMEM; Sigma-Aldrich Co., St Louis, MO, USA) containing $10 \%(\mathrm{v} / \mathrm{v})$ foetal bovine serum (FBS) at $37^{\circ} \mathrm{C}$ in a humidified $5 \% \mathrm{CO}_{2}$ atmosphere. Transfection of cells with plasmid DNA was performed using Lipofectamine 2000 (Invitrogen) according to the manufacturer's instructions.

Synthesis and purification of NBD-cPA. NBD-cPA was prepared from 12:0 NBDLPC as follows: NBD-LPC was dissolved in $10 \mathrm{mM}$ HEPES-NaOH (pH 7.9) and incubated with Actinomadura PLD at $37^{\circ} \mathrm{C}$ for $2 \mathrm{~h}$ to generate NBD-cPA through transphosphatidylation. Water-saturated butanol was added to stop the reaction and extract the lipids. TLC analysis of lipid extracts was performed on Merck Silica gel 60 HPTLC plates by developing with a mobile phase of chloroform:methanol:28\% ammonium hydroxide (6:4:1). NBD-cPA was visualised using a ChemiDoc XRS+ imager (Bio-Rad, Hercules, CA, USA), and the spot was scraped and extracted in chloroform:methanol (1:1). Purified NBD-cPA was then evaporated under argon and quantified by the molybdenum blue method ${ }^{43}$.

Preparation of cPA-hydrogel microspheres and analysis of cPA release. Gelatin hydrogel (MedGel ${ }^{\circledR}$-PI5 microsphere, $1 \mathrm{mg}$ ) was added to $20 \mu \mathrm{L}$ of phosphatebuffered saline (0.1 $\times$ PBS, pH 7.4) with or without $100 \mathrm{nmol}$ of $\mathrm{cPA}$ 17:0 (dissolved in DMSO), and was allowed to swell to equilibrium for $24 \mathrm{~h}$ at $4{ }^{\circ} \mathrm{C}$. The hydrogel matrices were then washed in PBS 5 times to remove excess $\mathrm{cPA}$. The amount of $\mathrm{CPA}$ encapsulated within the microsphere was quantified by the molybdenum blue method. HT- 29 cells were plated at $2 \times 10^{4}$ cells/well in $0.5 \mathrm{~mL}$ of DMEM-1\% FBS in a 24-well plate and incubated at $37^{\circ} \mathrm{C}$ for $12 \mathrm{~h}$. Hydrogel matrices were then added to the wells in $0.5 \mathrm{~mL}$ of DMEM-1\% FBS, and incubation was continued at $37^{\circ} \mathrm{C}$ for the indicated times. Cells were harvested by scraping, and lipids were extracted using the method reported by Bligh and Dyer ${ }^{44}$. The extracted lipids were dissolved in $0.1 \mathrm{~mL}$ of methanol:chloroform:28\% $\mathrm{NH}_{4} \mathrm{OH}$ (90:10:1) and immediately analysed by ESI-LCMS as previously described ${ }^{13}$. 


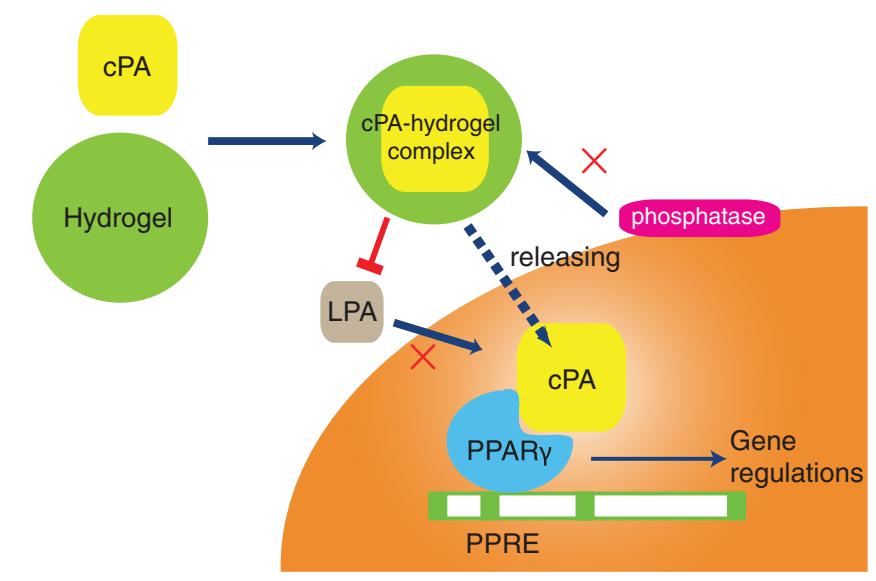

Figure 5 | Graphical representation of the release of cPA from hydrogel microspheres for intracellular delivery. (a) In the tested cell lines, lipid phosphate phosphatases (intracellular or extracellular) lead to the formation of LPA by hydrolytic cleavage of the cyclic phosphate ring of cPA. LPA-induced PPAR $\gamma$ activity was efficiently suppressed by cotreatment with cPA-hydrogel microspheres. Hydrogel microspheres are thus essential to prevent the degradation of cPA to LPA.

Measurement of PPAR $\gamma$ activity by a luciferase reporter assay. To determine PPAR $\gamma$ activation, HT-29 cells were co-transfected with the pcDNA3.1-PPAR $\gamma$ expression plasmid and the pGL3b-PPRE-Luc and pSV40- $\beta$-galactosidase reporter plasmids and analysed in a luciferase reporter assay, as previously described ${ }^{29}$. Twenty-four hours after transfection, cells were treated with vehicle (hydrogel), cPA, LPA, or CPA-hydrogel microspheres in DMEM without FBS and cultured for an additional $20 \mathrm{~h}$. Luciferase activity was measured with the Bright-Glo Luciferase Assay System (Promega, Madison, WI, USA) using a SpectraMax plate reader (Molecular Devices, Sunnyvale, CA, USA). The results shown are representative data from 3 independent experiments. Both activities were measured according to the manufacturer's protocol.

Effect of hydrogel-released cPA on cell proliferation. HT-29 cells were plated at $1 \times$ $10^{4}$ cells/well in a 96-well plate. Growth medium was removed and replaced with fresh serum-free DMEM containing the indicated concentrations of hydrogel and/or free cPA. Cells were then incubated for 2 days at $37^{\circ} \mathrm{C}$. To measure cell viability, $10 \mu \mathrm{L}$ of alamarBlue reagent (Invitrogen, Carlsbad, CA, USA) was added to each well, and the assay was performed according to the manufacturer's instructions. In this assay, the substrate is actively metabolised to the coloured water-soluble product, which is excreted into the culture medium and is measured as the change in absorbance at $570 \mathrm{~nm}$. The assay measures metabolic activity as an indicator of cell proliferation and/or cell viability.

Wound healing assay. Cell migration assays were performed using $\mu$-Dish 35-mm culture inserts (Ibidi, Martinsried, Germany) according to the manufacturer's protocols. In brief, HT-29 cells were seeded into each well of the culture inserts and incubated at $37^{\circ} \mathrm{C}$. After the cells were attached, the culture inserts were gently removed, and the cells were treated with fresh DMEM containing 0.1\% FBS along with the vehicle, $\mathrm{cPA}$, or cPA-hydrogel microspheres. Immediately after removing the inserts $(t=0)$ and at 48 and $72 \mathrm{~h}$, cultures were photographed by phase contrast microscopy, the width was determined, and the mean percent closure was calculated for each culture condition.

Modified boyden chamber cell invasion assay. Cells were seeded in 24-well plates at $5 \times 10^{4}$ cells/well, containing a polycarbonate filter with $8-\mu \mathrm{m}$ pores (Chemotaxicell CH8-24, Kurabo, Osaka, Japan) with $200 \mu \mathrm{L}$ of serum-free DMEM (Nacalai, Kyoto, Japan) and incubated for $6 \mathrm{~h}$ at $37^{\circ} \mathrm{C}$ in the presence of $3 \mu \mathrm{M} \mathrm{LPA}$ with or without $\mathrm{cPA}(500 \mu \mathrm{L})$ in the lower side of the filter. All non-migrant cells were removed from the upper face of the membrane with a cotton swab, whereas migrant cells on the lower face were fixed with absolute methanol and stained with Giemsa stain solution (Wako, Osaka, Japan). The number of cells that had migrated to the lower side of the filter was manually counted under a microscope. At least 4 fields of cells per sample were counted and tabulated.

Statistical analysis. Results were analysed by one-way Student's $t$-test and are expressed as mean \pm s.e.m. $P$ values less than 0.05 were considered statistically significant.

1. Denlinger, C. S. \& Engstrom, P. F. Colorectal cancer survivorship: movement matters. Cancer Prev. Res. (Phila) 4, 502-511 (2011).
2. Hurwitz, H. et al. Bevacizumab plus irinotecan, fluorouracil, and leucovorin for metastatic colorectal cancer. N. Engl. J. Med. 350, 2335-2342 (2004).

3. Tol, J. et al. Chemotherapy, bevacizumab, and cetuximab in metastatic colorectal cancer. N. Engl. J. Med. 360, 563-572 (2009).

4. Schafer, C., Schott, M., Brandl, F., Neidhart, S. \& Carle, R. Identification and quantification of epsilon-(gamma-glutamyl)lysine in digests of enzymatically cross-linked leguminous proteins by high-performance liquid chromatographyelectrospray ionization mass spectrometry (HPLC-ESI-MS). J. Agric. Food Chem. 53, 2830-2837 (2005).

5. Burton, J. D., Goldenberg, D. M. \& Blumenthal, R. D. Potential of peroxisome proliferator-activated receptor gamma antagonist compounds as therapeutic agents for a wide range of cancer types. PPAR Res. 2008, 494161 (2008).

6. Zaytseva, Y. Y., Wallis, N. K., Southard, R. C. \& Kilgore, M. W. The PPARgamma antagonist T0070907 suppresses breast cancer cell proliferation and motility via both PPARgamma-dependent and -independent mechanisms. Anticancer Res. 31, 813-823 (2011).

7. Evans, R. M. The nuclear receptor superfamily: A Rosetta Stone for physiology. Mol. Endocrinol. 19, 1429-1438 (2005).

8. Tontonoz, P. \& Spiegelman, B. M. Fat and beyond: the diverse biology of PPARgamma. Annu. Rev. Biochem. 77, 289-312 (2008).

9. Lim, S. et al. PPARgamma gene transfer sustains apoptosis, inhibits vascular smooth muscle cell proliferation, and reduces neointima formation after balloon injury in rats. Arterioscler. Thromb. Vasc. Biol. 26, 808-813 (2006).

10. Duval, C., Chinetti, G., Trottein, F., Fruchart, J. C. \& Staels, B. The role of PPARs in atherosclerosis. Trends. Mol. Med. 8, 422-430 (2002).

11. Yu, C. et al. The nuclear receptor corepressors NCoR and SMRT decrease peroxisome proliferator-activated receptor gamma transcriptional activity and repress 3T3-L1 adipogenesis. J. Biol. Chem. 280, 13600-13605 (2005).

12. Hawk, E. T., Umar, A. \& Viner, J. L. Colorectal cancer chemoprevention--an overview of the science. Gastroenterology 126, 1423-1447 (2004).

13. Tsukahara, T., Hanazawa, S., Kobayashi, T., Iwamoto, Y. \& Murakami-Murofushi, K. Cyclic phosphatidic acid decreases proliferation and survival of colon cancer cells by inhibiting peroxisome proliferator-activated receptor gamma. Prostaglandins Other Lipid Mediat. 93, 126-133 (2010).

14. Tsukahara, T. et al. Phospholipase D2-dependent inhibition of the nuclear hormone receptor PPARgamma by cyclic phosphatidic acid. Mol. Cell 39, 421-432 (2010).

15. Murakami-Murofushi, K. et al. A novel lipid mediator, cyclic phosphatidic acid (cPA), and its biological functions. Ann. N. Y. Acad. Sci. 905, 319-321 (2000).

16. Fujiwara, Y. Cyclic phosphatidic acid - a unique bioactive phospholipid. Biochim. Biophys. Acta 1781, 519-524 (2008).

17. Fujiwara, Y. et al. Identification of residues responsible for ligand recognition and regioisomeric selectivity of lysophosphatidic acid receptors expressed in mammalian cells. J. Biol. Chem. 280, 35038-35050 (2005).

18. Murakami-Murofushi, K. et al. Biological functions of a novel lipid mediator, cyclic phosphatidic acid. Biochim. Biophys. Acta 1582, 1-7 (2002).

19. Uchiyama, A. et al. Inhibition of transcellular tumor cell migration and metastasis by novel carba-derivatives of cyclic phosphatidic acid. Biochim. Biophys. Acta 1771, 103-112 (2007).

20. Mills, G. B. \& Moolenaar, W. H. The emerging role of lysophosphatidic acid in cancer. Nat. Rev. Cancer 3, 582-591 (2003).

21. Xu, Y. et al. Lysophosphatidic acid as a potential biomarker for ovarian and other gynecologic cancers. JAMA. 280, 719-723 (1998).

22. Lee, S. J. \& Yun, C. C. Colorectal cancer cells - Proliferation, survival and invasion by lysophosphatidic acid. Int. J. Biochem. Cell Biol. 42, 1907-1910 (2010).

23. Sun, H. et al. Effects of lysophosphatidic acid on human colon cancer cells and its mechanisms of action. World J. Gastroenterol. 15, 4547-4555 (2009).

24. Fukushima, N., Kimura, Y. \& Chun, J. A single receptor encoded by vzg-1/lpA1/ edg- 2 couples to $G$ proteins and mediates multiple cellular responses to lysophosphatidic acid. Proc. Natl. Acad. Sci. U. S. A. 95, 6151-6156 (1998).

25. Contos, J. J. et al. Characterization of lpa(2) (Edg4) and lpa(1)/lpa(2) (Edg2/Edg4) lysophosphatidic acid receptor knockout mice: signaling deficits without obvious phenotypic abnormality attributable to lpa(2). Mol. Cell. Biol. 22, 6921-6929 (2002).

26. Noguchi, K., Ishii, S. \& Shimizu, T. Identification of p2y9/GPR23 as a novel G protein-coupled receptor for lysophosphatidic acid, structurally distant from the Edg family. J. Biol. Chem. 278, 25600-25606 (2003).

27. Yamada, T. et al. Lysophosphatidic acid (LPA) in malignant ascites stimulates motility of human pancreatic cancer cells through LPA1. J. Biol. Chem. 279, 6595-6605 (2004)

28. McIntyre, T. M. et al. Identification of an intracellular receptor for lysophosphatidic acid (LPA): LPA is a transcellular PPARgamma agonist. Proc. Natl. Acad. Sci. U. S. A. 100, 131-136 (2003).

29. Tsukahara, T. et al. Different residues mediate recognition of 1-Ooleyllysophosphatidic acid and rosiglitazone in the ligand binding domain of peroxisome proliferator-activated receptor gamma. J. Biol. Chem. 281, 3398-3407 (2006).

30. Tsukahara, T. et al. Phospholipase D2-dependent inhibition of the nuclear hormone receptor PPARgamma by cyclic phosphatidic acid. Mol. Cell 39, 421-432 (2010).

31. Tigyi, G. Aiming drug discovery at lysophosphatidic acid targets. Br. J. Pharmacol. 161, 241-270 (2010). 
32. Fischer, D. J. et al. Naturally occurring analogs of lysophosphatidic acid elicit different cellular responses through selective activation of multiple receptor subtypes. Mol. Pharmacol. 54, 979-988 (1998).

33. Mukai, M. et al. Inhibition of tumor invasion and metastasis by a novel lysophosphatidic acid (cyclic LPA). Int. J. Cancer 81, 918-922 (1999).

34. Ishihara, R. et al. Attenuation by cyclic phosphatidic acid of peritoneal metastasis of azoxymethane-induced intestinal cancers in Wistar rats. Int. J. Cancer 110, 188-193 (2004).

35. Mukai, M. et al. Hepatoma cell migration through a mesothelial cell monolayer is inhibited by cyclic AMP-elevating agents via a Rho-dependent pathway. FEBS Lett. 484, 69-73 (2000)

36. Weng, L., Le, H. C., Lin, J. \& Golzarian, J. Doxorubicin loading and eluting characteristics of bioresorbable hydrogel microspheres: in vitro study. Int. J. Pharm. 409, 185-193 (2011).

37. Lee, P. I. \& Kim, C. J. Probing the mechanisms of drug release from hydrogels. J. Control. Release 16, 229-236 (1991).

38. Einerson, N. J., Stevens, K. R. \& Kao, W. J. Synthesis and physicochemical analysis of gelatin-based hydrogels for drug carrier matrices. Biomaterials 24, 509-523 (2003).

39. Mukherjee, S., Raghuraman, H., Dasgupta, S. \& Chattopadhyay, A. Organization and dynamics of N-(7-nitrobenz-2-oxa-1,3-diazol-4-yl)-labeled lipids: a fluorescence approach. Chem. Phys. Lipids 127, 91-101 (2004).

40. Fang, X. et al. Lysophosphatidic acid is a bioactive mediator in ovarian cancer. Biochim. Biophys. Acta 1582, 257-264 (2002).

41. Beerling, E., Ritsma, L., Vrisekoop, N., Derksen, P. W. \& van Rheenen, J. Intravital microscopy: new insights into metastasis of tumors. J. Cell Sci. 124, 299-310 (2011).

42. Tsukahara, T. The role of PPARgamma in the transcriptional control by agonists and antagonists. PPAR Res. 2012, 362361 (2012).
43. Lowry, R. R. \& Tinsley, I. J. A simple, sensitive method for lipid phosphorus. Lipids 9, 491-492 (1974)

44. Bligh, E. G. \& Dyer, W. J. A rapid method of total lipid extraction and purification. Can. J. Biochem. Physiol. 37, 911-917 (1959).

\section{Acknowledgements}

This work was supported by grants from the Astellas Foundation for Research on Metabolic Disorders (to Tamotsu Tsukahara) and the Takeda Science Foundation (to Tamotsu Tsukahara) and by a Grant-in-Aid for Scientific Research (C) 22591482 (to Tamotsu Tsukahara) from the Japan Society for the Promotion of Science (JSPS).

\section{Author contributions}

TT and KM conceived and designed the experiments. TT performed the experiments. TT analysed the data and wrote the paper.

\section{Additional information}

Competing financial interests: The authors declare no competing financial interests.

License: This work is licensed under a Creative Commons

Attribution-NonCommercial-ShareAlike 3.0 Unported License. To view a copy of this license, visit http://creativecommons.org/licenses/by-nc-sa/3.0/

How to cite this article: Tsukahara, T. \& Murakami-Murofushi, K. Release of Cyclic Phosphatidic Acid from Gelatin-based Hydrogels Inhibit Colon Cancer Cell Growth and Migration. Sci. Rep. 2, 687; DOI:10.1038/srep00687 (2012). 